\title{
Un aperçu «échologique» des sciences neurologiques ultramontaines
}

\author{
Louis J. Poirier \\ Centre de recherche en neurobiologie \\ Hôpital de l'enfant-Jesus, Québec
}

En premier lieu, je tiens à féliciter les principaux responsables de l'organisation de cette première journée des sciences neurologiques de l'Université de Montréal, soit les docteurs Guy Bouvier, Pierre Duquette, Yves Lamarre, Gérard Mohr, Pedro Molina-Negro et Serge Rossignol. Grâce à eux, à Madame Hélène Auzat, secrétaire, et à bien d'autres, cette journée s'est avérée un franc succès.

À l'occasion de cette conférence, je veux tenter de me faire l'écho des pionniers de la recherche en neuroscience à l'Université de Montréal, qui à cette époque avaient pour objectif de devenir les émules des chercheurs anglophones d'outremontagne. travaux faisant aussi appel à la cytogénèse et à l'histogénèse furent réalisés chez des larves ou embryons d'Amia calva de 2 à $21 \mathrm{~mm}$ de long. Mentionnons que les coupes utilisées à cette fin étaient colorées selon la méthode trichromique de Masson, alors directeur du Département de pathologie. À ce sujet Bonin affirme et je cite, Cette méthode s'est avérée d'une utilité incontestable en démontrant les vitrées de façon précise. ${ }^{3}$ II était donc très clairvoyant! De plus, Bonin a profité dans ses travaux de l'expertise de deux autres membres du Département de pathologie, soit celle de Louis-Charles Simard sur le plan microphotographique et Georges Manseau sur le plan dessin et à ce sujet, il faut voir les magnifiques planches qui appuient les

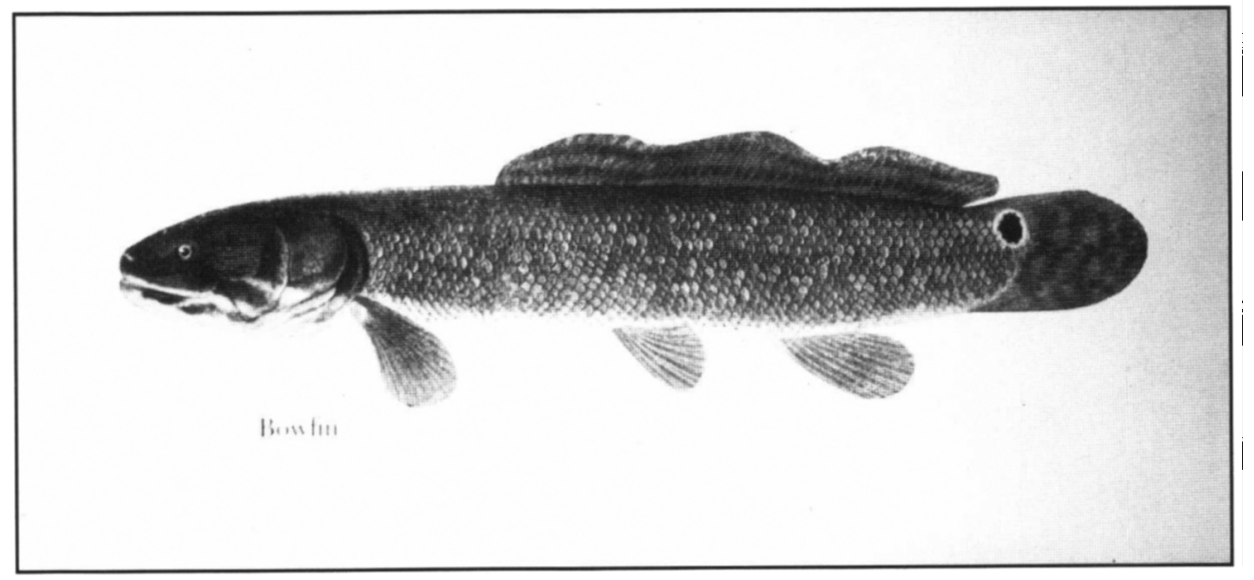

Cette photographie (Figure 1) tirée de Hubbs et Lagler' représente un poisson qui sans doute vous est très familier puisqu'il vit dans les baies marécageuses et qu'on peut le capturer à la foëne - soit Amia Calva. 'Il est habituellement classé parmi les ganoides - à squelette cartilagineux. Sa nageoire dorsale est arquée, d'où son nom anglais de Bowfin. Ce poisson est considéré comme un fossile vivant - puisqu'il est le seul survivant de sa famille - ses congénères étant disparus depuis des milliers d'années.

Or ce spécimen rare est, selon toute évidence, à l'origine de la recherche en neuroscience à l'université de Montréal. En effet, Wilbrod Bonin rattaché au Laboratoire d'histologie et d'embryologie, eut la main heureuse en arrêtant son choix sur Amia calva pour entreprendre des recherches à caractère embryologique et phylogénétique entre les années 1937-41.2-4 Ces descriptions de ces travaux. ${ }^{3,4}$ Ici je me permets un a parte et je vous avoue qu'un des avantages de la retraite c'est d'avoir le temps d'apprécier les travaux d'autrui et peut-être de mieux les jauger.

Ces travaux de Bonin à la fin des années ' 30 correspondent à cette période où l'anatomie comparée du système nerveux avec Herrick, Kuypers, Ziehen, Castaldi, Cajal, Huber, Rasmussen, Crosby, etc. connut un grand essor. Grâce à son matériel de recherche tout à fait approprié pour ce genre d'étude, Bonin réussit à individualiser chez Amia Calva le nerf terminal et son ganglion (le ganglion de Locy). Car à cette époque, certains mettaient en doute l'existence même de ce nerf crânien et de son ganglion alors que d'autres argumentaient relativement à l'origine et aux fonctions de ces structures. C'est ainsi que Bonin démontra l'importance primordiale de la placode épiblas- 
tique olfactive dans le développement du ganglion de Locy et du nerf terminal, aussi appelé le nerf \#0.3.4 C'est donc ici que débute une recherche originale en neuroscience à l'Université de Montréal. L'on peut donc affirmer que l'on est parti de zéro! Selon toute évidence, ce nerf innerve le neuroépithélium (olfactif) au niveau du septum médian des fosses nasales. Il a donc une très grande valeur sentimentale!

Alors que Bonin poursuivait ces travaux, Masson et ses jeunes collègues, Louis-Charles Simard, Georges Manseau et Joseph-Luc Riopelle s'intéressaient davantage aux structures nerveuses périphériques. Masson était déjà reconnu pour ses qualités d'expert et d'artiste dans la mise au point de techniques de coloration et d'imprégnation argentaffine à telle enseigne que ses collègues américains le qualifiaient de Grand teinturier. Donc à la même époque, ce groupe de pathologistes démontrèrent la présence de cellules argentaffines au niveau des plexus myentériques et sous-muqueux d'Auerbach et de Meissner et des complexes neuro-insulaires du pancréas qu'ils furent les premiers à décrire.

Quelques années plus tard, Eugène Robillard initiait des recherches à caractère neurophysiologique. En particulier, il était un ardent défenseur de la théorie du physiologiste français Louis Lapicque, théorie mettant en cause la chronaxie et la rhéobase. J'ai cru utile pour les moins avertis d'entre vous de rappeler la définition de ces deux termes. En effet, la rhéobase est l'intensité minimale nécessaire pour provoquer l'excitation d'un nerf ou d'un muscle alors que la chronaxie est le temps de passage du courant électrique nécessaire pour obtenir le seuil de contraction avec une intensité double de l'intensité liminaire (ou rhéobase). Comme vous voyez c'est éminemment simple et pratique! Aussi nos maîtres pouvaient affirmer dans ce temps tout comme vous aujourd'hui: beaucoup de notions ne sont plus admises qui semblaient acquises et incontestées. ${ }^{3}$

C'est donc dans ce contexte que j'eus le privilège de faire mes études médicales. Or je puis vous dire qu'en ces temps où la connaissance primait sur la pédagogie, l'étudiant ne pouvait se permettre, à l'occasion d'un examen oral, d'ignorer l'existence du nerf crânien \#0 et du ganglion correspondant de Locy, ou encore, de mettre en doute l'origine épiblastique de ces structures, sans quoi son avenir médical était sérieusement compromis. De même tout candidat qui confondait l'intensité minimale de la chronaxie avec l'intensité liminale de la rhéobase ou encore, mettait en doute la théorie de Lapicque à ce sujet avait un avenir hypocratique très sombre. En pathologie, une attitude distante à l'endroit des complexes neuro-insulaires ou des cellules argentaffines pouvait nuire grandement aux visées argentophiles ultérieures de tout disciple d'Esculape en puissance. J'allais oublier. À ce temps la recherche en anatomie macroscopique en était presque au point mort! Par contre ici l'on compensait par une pédagogie appropriée. C'est ainsi qu'en l'absence d'approches purement scientifiques, l'on privilégiait l'exercise mnémotechnique en sus du culte du maître à penser. C'est ainsi que, selon l'examinateur en devoir, le candidat, quantité négligeable, devait faire preuve de perspicacité. Car il pouvait aussi bien être invité à décliner avec une certaine pudeur toutes les ramifications de la branche externe du nerf honteux interne, ou à l'opposé, à divulguer avec emphase tous les insinuosités relatives à l'innervation du mammelon et les méandres de la mammaire profonde. J'espère que cette partie de mon exposé ne vous amènera pas à conclure que je suis devenu plus terre à terre que Sol.

Au plan clinique, c'était encore la neuropsychiatrie qui était à la mode. Entre autres, il y avait Roma Amyot, Antonio Barbeau, Jean Saucier et Émile Legrand, Amyot ćtant plus psychologiquement neurologue et Legrand plus organiquement psychiatre. Ces quatre cliniciens, tous formés à l'École française, avaient une remarquable culture générale et leur maîtrise de la complexe terminologie neurologique et psychiatrique de l'époque en faisait des érudits et des maîtres incontestés aux yeux de leurs collègues. Déjà à cette époque la neuroophtalmologie qui, par la suite, est devenue et est demeurée très avant-gardiste dans notre milieu, manifestait quelqu'originalité. En effet, un certain professeur se taxait d'avoir réalisé une étude en profondeur et de haut niveau alors que, selon ses dires, il fut le premier à procéder à un examen neuroophtalmologique de fond de l'oeil chez la girafe. Ce qui l'amenait à conclure humblement: Ici s'arrête la science et moi je continue.

Voilà résumé très succinctement l'atmosphère qui prévalait à la Faculté lorsqu'une nouvelle génération de chercheurs initièrent des recherches en sciences neurologiques fondamentales. En plus de votre invité oeuvrant en neuroanatomie et en neurologie expérimentale, il me faut nommer Jean-Jacques Lussier dont l'expertise impliquait l'électrophysiologie de la fibre nerveuse périphérique et Antoine D'lorio auquel s'est joint Jean Leduc ultérieurement. D'Iorio s'intéressait dans le sens large aux neurohumeurs et comme je ne connais d'humeurs qui n'échappent au contrôle du cerveau, D'lorio était un neuroscientiste. À ce moment, il s'intéressait, entre autres choses, aux granules de la médullosurrénale en tant que témoins de la libération des catécholamines. ${ }^{5}$ Il était loin de se douter que ses travaux auraient des implications dans la récupération fonctionnelle du système extrapyramidal associé aux greffons surrénaliens intracérébraux. Par la suite, Lussier est devenu doyen de la Faculté de médecine de l'université d'Ottawa alors que D'Iorio en est présentement le recteur. Ces deux chercheurs recrutés par Eugène Robillard ont contribué de façon manifeste au rayonnement de leur alma mater. Quant à moi, recruté par Wilbrod Bonin, quel témoignage de reconnaissance pourrait m'être plus cher que celui d'être conférencier invité à l'occasion de cette "Première Journée des Sciences Neurologiques". En effet, c'est une trés élégante façon de reconnaître ma contribution à l'essor des neurosciences à l'université de Montréal où l'on retrouve maintenant en si grand nombre des scientistes à la fine pointe de la substance cérébrale en activité.

Très tôt se joignaient à nous Jean-Pierre Cordeau, ncurophysiologiste, et Aurèle Beaulnes, ce dernier s'intéressant entre autres, à la neuropharmacologie. À son tour Cordeau attirait Hélène Mahut, psychologue expérimentale, Jacques Courville devenu neuroanatomiste et Léo-Yves Lamarre, neurophysiologiste dont les connaissances, le charisme et le leadership ne sont pas étrangers aux succès du plus important groupe de neuroscientistes canadiens. Jean-Pierre Raynaud, physicien et électrophysiologiste, et Jacques de Champlain, versé dans les neurohumeurs cliniques et expérimentales, font aussi partie de l'équipe de la première heure. En même temps se joignaient à moi Eva Shulman, Jean-Louis Lalonde, Claude Gauthier, Robert Ayotte, Anne-Marie Mouren-Mathieu et Claude-Lise Richer tous collaborateurs et collaboratrices de la première 
heure. Ȧ la même période, Léon Tétreault en collaboration avec Jean-Marc Bordeleau initiaient des recherches en neuropsychopharmacologie où l'approche statistique devenait exceptionnellement de rigueur. Or l'ensemble des travaux réalisés à cette époque ont été soulignés par l'Union Médicale qui a consacré un numéro complet au Laboratoire des sciences neurologiques en $1961^{6}$ (préfacé) par Roma Amyot. ${ }^{7}$ De même, un important colloque portant sur le Système extrapyramidal et les neuroleptiques dont les compte-rendus ont paru dans la Revue Canadienne de Biologie 8 reflète aussi les activités scientifiques de plusieurs chercheurs oeuvrant dans les sciences neurologiques à l'Université de Montréal à cette période.

Si l'on revient au groupe-clé en neuroscience, il nous faut mentionner les noms de Herbert Jasper et Marc Colonnier, chercheurs chevronnés, dont la venue n'est pas non plus étrangère à la qualité des nombreuses réalisations scientifiques de ce groupe. En raison de l'essor considérable qu'a connu ce groupe ainsi que les chercheurs cliniciens, je me trouve dans l'obligation d'arrêter ici l'identification des branches de plus en plus fines de cet arbre généalogique tellement elles sont nombreuses. Ce qui n'en amoindrit pas pour autant la qualité des fruits qu'elles portent.

Au plan clinique, la recherche en neuroscience a débuté avec de nouvelles générations de cliniciens. Claude Bertrand avec la collaboration ultérieure de Napoléon Martinez fut le premier à apporter une contribution scientifique importante en neurochirurgie et, en particulier, relativement au traitement neurochirurgical de la maladie de Parkinson. Jules Hardy se joignit bientôt à lui et à son tour fut reconnu pour ses travaux découlant d'approches à la fois neurophysiologique et chirurgicale relatives aux tumeurs hypophysaires. En neurologie, Guy Courtois, Claude Gauthier, Normand Giard et surtout André Barbeau donnèrent une nouvelle impulsion à la recherche dans ce secteur des neurosciences. En particulier, Barbeau contribua de façon substantielle à la mise au point du traitement de la maladie de Parkinson par la L-DOPA, comme on l'a si bien souligné durant cette journée.

Comme vous avez pu le déduire, la contribution des chercheurs de l'Université de Montréal à la compréhension et à la thérapie de troubles extrapyramidaux se situe à trois palliers différents d'expertise: en sciences fondamentales, en neurochirurgie et en neurologie et je pourrais même ajouter en neuropsychopharmacologie avec l'arrivée des neuroleptiques. Tout ceci n'est pas un effet du hasard. Au contraire, il s'agit d'un phénomène d'interaction entre fondamentalistes et cliniciens à plusieurs niveaux. D'une part, nous collaborions avec l'équipe de Notre-Dame dans l'identification des voies de la douleur ${ }^{9}$ et dans la mise au point d'une technique radiostéréotaxique et électrophysiologique.

Cette dernière avait pour but le repérage précis de la cible idéale à atteindre pour réduire et le tremblement et la rigidité chez le patient parkinsonien. ${ }^{10} \mathrm{D}$ 'autre part, la collaboration des neurochirurgiens nous était assurée dans l'application de techniques chirurgicales destinées à induire les troubles moteurs associés au parkinsonisme chez le singe, ces travaux étant destinés à mettre en évidence les mécanismes nerveux en cause. Et ces recherches reprises par nos successeurs se continuent aujourd'hui avec de plus en plus de raffinements et de retombées scientifiques originales. Encore une fois, l'Union Médicale a tenu à souligner l'importante contribution des chercheurs de l'Université de Montréal dans le domaine des sciences neurologiques en y consacrant un numéro complet en 1985." En particulier, l'on y retrouve un excellent résumé historique d'Yves Lamarre. ${ }^{12}$

Par ailleurs une non moins fructueuse collaboration s'est instaurée entre les neuroscientistes de McGill et ceux de Montréal. C'est ainsi que des singes rendus parkinsoniens à l'Université de Montréall3 servirent en 1960 à la mise en évidence et à la mesure d'activités unitaires anormales au niveau du cortex moteur. Ces expériences étaient réalisées par Jasper en collaboration avec Cordeau alors en stage post-doctoral à I'Institut neurologique. ${ }^{14} \mathrm{D}$ 'autre part, des cerveaux de singes porteurs de lésions intéressant aussi le système extrapyramidal (en particulier la substance noire) servirent à la mise en évidence de modifications importantes des monoamines cérébrales dans les structures nerveuses cibles. ${ }^{15}$ Les déterminations neurochimiques étaient faites par le docteur Sourkes de McGill qui a aussi collaboré à l'occasion avec André Barbeau relativement aux modifications monoaminergiques humorales chez les parkinsoniens. ${ }^{16}$

De même que nous assistions à une étroite collaboration entre les chercheurs de McGill et de Montréal, une collaboration non moins fructueuse s'est amorcée entre neuroscientistes français et montréalais autour de problématiques mettant en cause surtout le système extrapyramidal. C'est ainsi que Denise Albe-Fessard, en neurophysiologie, Gérard Guiot, en neurochirurgie et Pierre Deniker en neuropharmacologie ont été les pionniers du côté français à réaliser des travaux de collaboration avec leurs vis-à-vis du groupe des neurosciences de l'université de Montréal et de scientifiques cliniciens oeuvrant dans le réseau hospitalier, en particulier, Claude Bertrand et Jules Hardy en neurochirurgie, et Jean-Marc Bordeleau et Léon Tétreault en neuropharmacologie. Parmi les nombreux autres chercheurs français qui ont collaboré de façon étroite avec leurs collègues montréalais, il nous faut nommer Pierre Buser, Robert Naquet, Michel Jouvet, Jean Scherrer, Jean Massion, Pierre Rondot, Pierre Angaut, Jacques Glowinski, et bien d'autres. Du côté montréalais, il me faut nommer dans l'ordre chronologique votre conférencier, Jean-Pierre Cordeau, André Barbeau, Yves Lamarre, Laurent Descarries, Guy Bouvier, et j'en passe. L'on retrouve encore ici l'influence prépondérante de Herbert Jasper des deux côtés de l'Atlantique.

En guise de conclusion, il me fait grand plaisir d'affirmer à l'occasion de cette "Première Journée des Sciences Neurologiques", que tous et chacun appartenez à une communauté de neuroscientifiques très prestigieuse dont le rayonnement est considérable. Aussi, je formule le souhait que cette première soit le prétexte d'interactions de plus en plus nombreuses et étroites entre chercheurs cliniciens et fondamentalistes, ce qui se traduirait par des retombées scientifiques encore plus importantes à la fois pour l'avancement des connaissances en neurosciences et la qualité des soins qui en découle.

\section{BibliographIE}

1. Hubbs CC, Lagler KF. Fishes of Great Lakes Region. Cranbrook Institute of Science, Bloomfield Hills, Michigan 1947; 26. 
2. Bonin W. La contribution de l'épiblaste à la formation du ganglion acoustique et des ganglions annexés aux nerfs crâniens mixtes chez Amia calva. CR Soc Biol 1937; 124: 153-154.

3. Bonin W. Contribution à l'étude de l'origine du ganglion de Locy chez Amia calva. Arch de Biol 1939; 50: 321-342.

4. Bonin W. Étude anatomique du système nerveux périphérique et des organes des sens de la tête chez l'embryon d'Amia calva. Origine épiblastique des ganglions sensitifs crâniens. Contrib Inst Zool Univ Montréal 1940; 6: 1-62.

5. D'lorio A. The release of catecholamines from the isolated chromaffine granules of the adrenal medulla using sulphydryl inhibitors. Can J Biochem Physiol 1957; 35: 395-400.

6. L'Union Médicale du Canada. Numéro spécial dédié au Laboratoire des sciences neurologiques 1961; 90(8): 771-873.

7. Amyot R. Les sciences neurologiques. Union Méd Can 1961; 90: 771.

8. Symposium international sur le système extrapyramidal et les neuroleptiques. Montréal, Nov. 1960. Publié dans la Rev Canad Biol 1961; 20.

9. Poirier LJ, Bertrand C. Experimental and anatomical investigation of the lateral spinothalamic and spinotectal tracts. J Comp Neurol 1955; 102: 745-758.
10. Bertrand C, Martinez N, Poirier LJ, Gauthier C. Une méthode de traitement chirurgical de la maladie de Parkinson. Union Méd Can 1960; 89: 287-299.

11. L'Union Médicale du Canada. Numéro dédié au Centre de recherche en sciences neurologiques de l'Université de Montréal 1985; 114(12): 957-1037.

12. Lamarre $Y$. Le Centre de recherche en sciences neurologiques de l'Université de Montréal. Union Méd Can 1985; 114(12): 960962. Préface.

13. Poirier LJ. Experimental and histological study of midbrain dyskinesias. J Neurophysiol 1960; 23: 534-552.

14. Cordeau JP, Gybels J, Jasper HH, Poirier LJ. Microelectrode studies of unit discharges in the sensorimotor cortex: investigations in monkeys with experimental tremor. Neurology 1960; 10: 591600.

15. Poirier LJ, Sourkes TL. Influence of the substantia nigra on the catecholamine content of the striatum. Brain 1965; 88: 181-192.

16. Barbeau A, Sourkes TL, Murphy GF. Les catécholamines dans la maladie de Parkinson. Dans: "Monoamines et Système Nerveux Central". Ed J de Ajuriaguerra. Georg, Geneve et Masson, Paris; 1962: 247-262. 